\title{
Self-Narratives in Organizations: Transgender and Gender Non-conforming Experiences
}

\section{Editorial note*}

\author{
GIUSEPPE BALIRANO \\ gbalirano@unior.it
}

University of Naples "L'Orientale"

\author{
LUIGI MARIA SICCA \\ luigimaria.sicca@unina.it \\ University of Naples Federico II
}

\author{
PAOLO VALERIO \\ paolo.valerio@unina.it \\ University of Naples Federico II
}

This thematic issue of the puntOorg International Journal draws together contributions that contain, on the one hand, original multidisciplinary research offering a range of critical perspectives on different representations of gender in the context of recent and old conflicting discourses about transgender and gender non-conforming (TGNC) people. On the other hand, the issue collects self-narratives from TGNC individuals who narrate their stories about working in organizations or businesses as transgender or gender nonconforming people, including (but not limited to) issues around transitioning and discrimination.

One of the reasons that have led us as editors in coming up with this thematic issue is the specific challenges that TGNC people face both in the workplace (Law et al., 2011) and during the recruitment process (McFadden and Crowley-Henry, 2016). Even if most of the research carried out in the field of Diversity Management is mainly focused on recruitment (Thomas and Plaut, 2008), and despite the fact that the investigation of practices linked to TGNC people is considered as typically associated with those scholars who investigate the binary view in organizations (Schilt and Westbrook, 2009), very few empirical research has been carried out on forms of discrimination and stigma taking place at the time of recruitment. Furthermore, from a psychological point of view, there is evidence that challenges TGNC people experience in the workplaces affect well-being negatively,

\footnotetext{
${ }^{*}$ The editors would like to thank for their hard work and support in the development of this thematic issue Davide Bizjak (University of Naples Federico II), Antonio Fruttaldo (University of Naples "L'Orientale"), Cristiano Scandurra (University of Naples Federico II).
} 
increasing the risk of developing adverse health outcomes, such as stress, anxiety, or depression (Dispenza et al., 2012). On the other hand, previous studies (e.g. Brewster et al., 2014) highlight that TGNC people are also able to adopt adaptive coping strategies to face stigmatizing events occurring within the workplace. Notwithstanding these observations in the context of an organizational framework, even psychological studies addressing stigma, well-being, and resilience of TGNC people in the workplace are still scarce.

The editors' primary aim in creating this constellation of original work was to explicitly challenge the several constraints, prejudices, and stereotypes concerning gender variant people by encouraging further academic investigation and, eventually, social change through discussions relating to gender non-conforming individuals from multidisciplinary viewpoints.

The rigid or stereotyped notions of gender and sexuality continue to flourish in systems of knowledge, belief, and power relating to communities of queer, gender non-conforming and transgender people. It is no surprise that the emergence of such a contemporary gender variant trope may work to re-orientate questions of diverse or, rather, non-heteronormative sensibility, sexuality, and identity in both local and global contexts. Therefore, the emphasis on self-narratives in this thematic issue of the puntOorg International Journal represents a powerful, performative act that works to compensate for the lack of attention to fundamental issues in the lives of these individuals. In particular, TGNC individuals are often on the receiving end of discrimination and unfair treatment in the workplace, which can even escalate to violence or complete rejection. Davis (2009), for instance, has highlighted how many TGNC workers feel that their workplace is unsafe or inhospitable. Notwithstanding these difficulties, TGNC people are able to tap into significant personal and collective resources in order to protect themselves from the negative effects of discrimination (Law et al., 2011). In order to better understand the personal and work-related processes linked to being in and living the workplace as a TGNC individual, it is crucial to give voice directly to those who experience this reality. This is the reason why self-narratives and the role played by language in shaping reality and expressing the inner self-experiences play a seminal role and become a keystone to the understanding of how forms of discrimination are experienced by TGNC individuals. If narratives can be seen as a way through which identities are shaped, the aim of this collection of work is to critically investigate and discuss different aspects of being a TGNC individual and the social stigma experienced in everyday life and the workplace.

The editors have organized this collection of papers in two separate parts, each one with its own scope but all connected to the very same aim: focusing on TGNC narratives by discussing both other- and self-representations of gender identity as powerful instruments to enhance diversity and inclusion.

The first section presents original contributions on theoretical and methodological reflections by scholars coming from different disciplinary fields in order to review and discuss the various approaches to TGNC narratives. The contribution by Davide Bizjak (University of Naples Federico II) approaches issues related to TGNC people from an organizational perspective. More specifically, the aim of the author is to scrutinize to what extent the role of transgender people's narratives may foster inclusion in organizations thanks to an account of the underlying meanings in writing organizational stories. The reflection offered by Davide Bizjak is mainly based on two concepts: the first one is silence, as a way of hiding in the workplace, and the second one is identity, as an apparatus of comparison between individual and organizational subjectivity. 
Through the lens of the minority stress theory and by performing a thematic inductive analysis, the second contribution by Cristiano Scandurra (University of Naples Federico II) and Vincenzo Bochicchio (University of Calabria) explores the emotional and psychological implications of the gender transition process in the workplace in two male-to-female and six female-to-male Italian TGNC individuals taking part in the study Re-interpreting organizations through transgender and gender non-conforming narratives.

Angela Zottola (University of Naples Federico II) discusses the relevance of linguistic research with regards to transgender identities representation and self-narration in the third contribution belonging to the first section of the issue. The discussion also includes a qualitative Critical Discourse Analysis of the collection of seven narratives and testimonies of transgender individuals from Italy in relation to their workplace experience and their coming out stories that are part of the second section of this thematic issue.

Lexie Webster (Lancaster University) closes this first section by offering a corpus-based analysis of gender-variant discourse on the social networking platform Twitter, exploring users' strategies for organizing their experience and understanding of employment. In her contribution, an analysis of the corpus of users' tweets explores the activated discourses around aspects of employment.

The second section of this thematic issue of the puntOorg International Journal contains seven self-representational narratives provided by TGNC people on their work and life experiences. The translation into English from the original Italian texts were provided by seven MA students in Language and Translation from the University of Naples "L'Orientale".

In this thematic issue, we address how the implicit gender binarism in the workplaces impacts on the feeling of discrimination experienced by transgender and gender nonconforming people in organization. Moreover, we focus on that part of research that concerns people's workplace life and the interplay between individual identities and organizational identities. The contribution of the self-narratives has been paramount in the creation of this issue, due to the seminal role of empirical data and research contributions, the combination of which allows for a better understanding of power relations and ideologies in discourse. The editors hope this collection of essays on TGNC individuals may be a starting point for future attention and multidisciplinary investigation on cogent issues that need to be looked at with more detailed analysis, avoiding any possible form of unnecessary bias, in order to link personal internal experiences of gender identity with possible objects of empirical multidisciplinary research concerning the negotiation of transitioning identities in the workplace and its effects on psychological well-being, the deconstruction of binary conception of norms, discursive and social practices, and practices of inclusion.

\section{Reference list}

Brewster, M.E., Velez, B.L., Mennicke, A., Tebbe, E. (2014) “Voices from beyond: A thematic content analysis of transgender employees' workplace experiences", Psychology of Sexual Orientation and Gender Diversity, 1 (2): 159-169.

Davis, D. (2009) “Transgender Issues in the Workplace: HRD's Newest Challenge/Opportunity", Advances in Developing Human Resources, 11 (1): 109-120. 
Dispenza, F., Watson, L.B., Chung, Y.B., Brack, G. (2012) "Experience of Career-Related Discrimination for Female-To-Male Transgender Persons: A Qualitative Study", The Career Development Quarterly, 60 (1): 65-81.

Law, C.L., Martinez, L.R., Ruggs, E.N., Hebl, M.R., Akers, E. (2011) “Trans-Parency in the Workplace: How the Experiences of Transsexual Employees Can Be Improved", Journal of Vocational Behavior, 79 (3): 710-723.

McFadden, C., Crowley-Henry, M. (2016) A Systematic Literature Review on Trans* Careers and Workplace Experiences, In Sexual Orientation and Transgender Issues in Organizations: Global Perspectives on LGBT Workforce Diversity, T. Köllen (ed.), pp. 63-81, Heidelberg: Springer.

Schilt, K., Westbrook, L. (2009) "Doing Gender, Doing Heteronormativity: "Gender Normals," Transgender People, and the Social Maintenance of Heterosexuality", Gender \& Society, 23 (4): 440-464.

Thomas, K.M., Plaut, V.C. (2008) “The Many Faces of Diversity Resistance in the Workplace", In Diversity Resistance in Organizations, K.M. Thomas (ed.), pp. 1-22, New York/London: Lawrence Erlbaum Associates.

Brewster, M.E., Velez, B.L., Mennicke, A., Tebbe, E. (2014) “Voices from beyond: A thematic content analysis of transgender employees' workplace experiences", Psychology of Sexual Orientation and Gender Diversity, 1 (2): 159-169.

Davis, D. (2009) “Transgender Issues in the Workplace: HRD's Newest Challenge/Opportunity", Advances in Developing Human Resources, 11 (1): 109-120.

Dispenza, F., Watson, L.B., Chung, Y.B., Brack, G. (2012) "Experience of Career-Related Discrimination for Female-To-Male Transgender Persons: A Qualitative Study", The Career Development Quarterly, 60 (1): 65-81.

Law, C.L., Martinez, L.R., Ruggs, E.N., Hebl, M.R., Akers, E. (2011) “Trans-Parency in the Workplace: How the Experiences of Transsexual Employees Can Be Improved", Journal of Vocational Behavior, 79 (3): 710-723.

McFadden, C., Crowley-Henry, M. (2016) A Systematic Literature Review on Trans* Careers and Workplace Experiences, In Sexual Orientation and Transgender Issues in Organizations: Global Perspectives on LGBT Workforce Diversity, T. Köllen (ed.), pp. 63-81, Heidelberg: Springer.

Schilt, K., Westbrook, L. (2009) "Doing Gender, Doing Heteronormativity: "Gender Normals," Transgender People, and the Social Maintenance of Heterosexuality", Gender E Society, 23 (4): 440-464.

Thomas, K.M., Plaut, V.C. (2008) "The Many Faces of Diversity Resistance in the Workplace", In Diversity Resistance in Organizations, K.M. Thomas (ed.), pp. 1-22, New York/London: Lawrence Erlbaum Associates. 